\title{
COMPONENTES DOS MODELOS DE NEGÓCIO PARA IOT NA ÁREA DE DESENVOLVIMENTO DE PRODUTOS
}

Tatiana Domingues de Almeida (tatiana.almd@gmail.com) -Universidade Federal de Santa

Catarina

Diego de Castro Fettermann (d.fettermann@ufsc.br) -Universidade Federal de Santa Catarina

\section{RESUMO}

A incorporação de tecnologias denominadas de Internet das Coisas (Internet of Things - IoT) é crescente tanto no desenvolvimento de produtos e serviços. Na área de tecnologia, são frequentes os casos de negócios que são criados a partir do desenvolvimento de um produto. Para organizar o desenvolvimento desses novos negócios foram desenvolvidos os Modelos de Negócio (Business Models - BM), que podem ser criados seguindo diversos focos, inclusive o de tecnologias IoT.

A utilização deste tipo de tecnologia permite o atendimento de diversas oportunidades de novos produtos, entretanto sua aplicabilidade no desenvolvimento de novas empresas ainda é pouco explorada na literatura. Este artigo tem por objetivo realizar uma revisão de literatura sobre a estrutura dos Modelos de Negócios para desenvolvimento de produtos e serviços com tecnologias IoT. Como fonte de dados utilizou-se uma revisão sistemática da literatura na base de dados internacional Science Direct para a busca dos Modelos de Negócios para IoT. A partir da coleta de dados desenvolveu-se uma análise de conteúdo dos Modelos de Negócio para IoT, a fim de identificar as características gerais dos BM para IoT e os componentes mais frequentes utilizados neste tipo de BM.

A partir da coleta de dados espera-se desenvolver uma análise de conteúdo dos Modelos de Negócio para IoT, de modo a identificar os principais elementos analisados nos modelos para esse fim. Com os resultados alcançados apresenta-se como contribuição teórica: (i) identificação dos componentes dos Modelos de Negócio para IoT mais frequentes nas propostas, (ii) realização de um compêndio dos Modelos de Negócios direcionados à IoT. Entre as contribuições práticas dos resultados podemos mencionar a criação de base de dados para empresas que desejam empregar novos Modelos de Negócio para desenvolvimento dos seus produtos a partir de tecnologias IoT.

Palavras-chave: IoT; Modelos de Negócio, Internet das Coisas; Desenvolvimento de Produtos e Serviços; Inovação. 


\section{INTRODUÇÃO}

A Internet das Coisas (Internet of Things - IoT), também denominada de como Web of Things, Internet of Evertything e Industrial Internet (PACHECO; KLEIN; RIGHI, 2016), tem sido observada como um dos principais assuntos discutidos tanto nos setores empresariais como em trabalhos acadêmicos recentemente (JU; KIM; AHN, 2016). Este termo se refere à integração e interconexão entre diversos tipos de objetos através de variados tipos de sensores e conexões à rede, para que interajam entre si e com o meio e estejam aptos a entender, monitorar e até mesmo controlar o ambiente (BAKER et al., 2017; NG; WAKENSHAW, 2017). Estudos indicam que as aplicações de IoT são diversas, apresentando possibilidades de utilização desse tipo de tecnologias em setores como manufatura, saúde, seguros, transporte, educação, agricultura, comércio, entre outros (AL-FUQAHA et al., 2015). As tecnologias utilizadas no IoT são recentes, porém há pouco tempo apresentam relevância no ponto de vista dos negócios (PACHECO; KLEIN; RIGHI, 2016). Nesse contexto, a introdução de novas tecnologias tem apresentado oportunidades para novas proposições nos modelos de negócios (Business Models - BM) em setores como logística, manufatura e produção, automação industrial, manutenção, desenvolvimento de produtos e serviços, entre outros (GLOVA; SABOL; VAJDA, 2014). Sendo assim, a IoT pode agregar benefícios como otimização de processos, sistemas complexos autônomos e análise de decisão guiada por sensores (JU; KIM; AHN, 2016).

No contexto do desenvolvimento de novos produtos e serviços, para prosperar em seus processos e resultados as empresas e gerência devem obter inteligência para selecionar estratégias de seus novos produtos. Para esse fim, é importante realizar uma gestão eficaz dos novos processos de produto (Processo de Desenvolvimento de Produto - PDP), desde a ideia inicial até seu lançamento no mercado, envolvendo todo o ciclo do produto (COOPER; KLEINSCHMIDT, 1987). Para que um produto tenha sucesso, é importante que tenha proposições de valor concretas que atendam à uma lógica e configuração de fontes de receita que, nos últimos anos, estão associadas à inovação digital (PACHECO; KLEIN; RIGHI, 2016). Por este motivo, nos últimos anos os modelos de negócio inovadores vêm ganhando atenção do ponto de vista comercial nas empresas (HASHEM et al., 2016).

Como advento de inovação, a Internet das Coisas disponibiliza novos serviços e novas oportunidades de negócio, com a criação de valor em produtos e serviços. Sua natureza de interconexão guia as organizações à maior abertura e colaboração entre si, o que torna mais complexa a construção de seus modelos de negócio (JU; KIM; AHN, 2016). Neste contexto, as empresas precisam repensar estrategicamente seus atuais modelos de negócio, para serem capazes de dominar o mercado emergente de IoT. No entanto, muitas empresas e gestores têm dificuldades de usar IoT para desenvolver seus modelos de negócio, principalmente porque a dinâmica do mercado voltado a IoT não é explícita em modelos encontrados na literatura (HASHEM et al., 2016; JU; KIM; AHN, 2016)

Observa-se a partir de pesquisas em bases de dados acadêmicas que há interesse crescente nos modelos de negócio para produtos e serviços baseados em IoT, porém ainda há um vasto campo a ser investigado sobre o tema (PACHECO; KLEIN; RIGHI, 2016). Levando em consideração essas dificuldades, este artigo tem por objetivo realizar uma revisão de literatura sobre a contribuição dos Modelos de Negócios para o desenvolvimento de produtos e serviços com tecnologias IoT. A partir da coleta de dados espera-se desenvolver uma análise de conteúdo dos Modelos de Negócio para IoT, para identificar os principais elementos analisados nos modelos para esse fim. Com os resultados alcançados neste artigo espera-se apresentar como contribuição teórica: (i) identificação dos componentes dos Modelos de 
Negócio para IoT mais frequentes nas propostas, (ii) realização de um compêndio dos Modelos de Negócios direcionados à IoT. Entre as contribuições práticas dos resultados podemos mencionar a criação de base de dados para empresas que desejam empregar novos Modelos de Negócio para desenvolvimento dos seus produtos a partir de tecnologias IoT.

\section{INTERNET DAS COISAS (Internet of Things - IoT)}

A Internet das Coisas (IoT) pode ser conceituada como uma infraestrutura global que disponibiliza serviços avançados através da interconexão física e virtual de objetos com base na utilização de tecnologias que realizam a comunicação e o processamento de informações (ATZORI; IERA; MORABITO, 2010). Como definição mais aplicada, IoT pode ser considerada uma conectividade inteligente de uma rede de dispositivos físicos utilizados para agregar resultados consideráveis em eficiência, crescimento do negócio e qualidade de vida das pessoas (NORONHA et al., 2014).

Para que haja a interconexão e interação dos produtos na rede, é necessário o uso de algumas tecnologias, inseridas em subsistemas. Alguns dos subsistemas existentes na arquitetura IoT são: identificação, monitoramento, rede de comunicação, computação, aplicativo, serviços e semântica (AL-FUQAHA et al., 2015; MOURTZIS et al., 2016). O uso dessa arquitetura apresenta variações dependendo do intuito de aplicação e dos resultados esperados. Dentre as principais tecnologias encontradas na literatura para desenvolvimento de produtos e serviços com incorporação de IoT destacam-se Radio Frequency Identification RFID (ATZORI; IERA; MORABITO, 2010; BORGIA, 2014; JU; KIM; AHN, 2016; PACHECO; KLEIN; RIGHI, 2016), sensores inteligentes (BORGIA, 2014; JU; KIM; AHN, 2016; PACHECO; KLEIN; RIGHI, 2016), dispositivos móveis (BORGIA, 2014; PACHECO; KLEIN; RIGHI, 2016) e Wireless Sensor Networks (WSNs) (BORGIA, 2014; GUBBI et al., 2013; HASHEM et al., 2016).

A aplicação de tecnologias IoT nas empresas, na indústria e no dia-a-dia das pessoas pode causar uma transformação no modo de vida dos consumidores e nos processos gerenciais e industriais (JU; KIM; AHN, 2016; NG; WAKENSHAW, 2017; PANG et al., 2015). O uso dessas tecnologias pode trazer benefícios ao meio ambiente, à sociedade, aos indivíduos e aos negócios a partir da criação de novas aplicações inteligentes e do desenvolvimento de novos produtos e serviços em domínios simultâneos que garantem a proteção e privacidade na troca de conteúdos e informações (BORGIA, 2014).

\section{MODELOS DE NEGÓCIO (Business Models - BM)}

A origem dos modelos de negócio na literatura deu-se por volta do final da década de 1990, quando se verificou um maior interesse de acadêmicos e profissionais em abordar o tema em variadas áreas de pesquisa (PACHECO; KLEIN; RIGHI, 2016). Diversas definições sobre modelos de negócio são encontradas na literatura. Na concepção de Zott e Amit (2010), os modelos de negócio são totalmente voltados para a criação de valor a todas as partes envolvidas; são representados pelos componentes, estrutura e governança de transações efetivadas. A partir disso, o modelo de negócio pode ser capaz de criar valor por meio da exploração de oportunidades de negócios (ZOTT; AMIT, 2010).

Na visão de Osterwalder, Pigneur e Tucci (2005), um modelo de negócio é uma ferramenta conceitual que engloba um conjunto de objetos, conceitos e suas respectivas relações, objetivando expressar a lógica por trás dos negócios de uma empresa. Os autores ressaltam que é necessário levar em consideração quais conceitos e relações são capazes de 
efetivamente representar o valor que é entregue ao cliente (OSTERWALDER; PIGNEUR; TUCCI, 2005).

Como abordagem mais ampla, os modelos de negócio podem ser apresentados como uma estrutura multidimensional composta por dimensões tecnológicas, dimensões industriais, dimensões políticas e dimensões estratégicas (LI; XU, 2013). Como definições voltadas ao cliente os modelos de negócio são conceituados como importância da informação como maior fonte de criação de valor e proposição de valor (UCKELMANN; HARRISON; MICHAHELLES, 2011) e uma estratégia centrada no cliente, através de compartilhamento de informações e integração de recursos (QIN; YU, 2015).

Em uma definição mais prática, os modelos de negócio podem ser entendidos como um modelo tridimensional que envolve colaboradores, redes de contato, ações táticas, entradas, serviços/processamento/embalagem, benefícios, estratégias e conteúdo/informações de produto (CHAN, 2015). E como conceito mais voltado à IoT, pode-se entender que é uma construção de blocos que são relevantes em IoT e identificação da importância da construção desses blocos (DIJKMAN et al., 2015). Esse último conceito está ligado a um específico modelo de negócio, conhecido como Business Model Canvas. Essa ferramenta foi desenvolvida por Osterwalder e Pigneur (2010) e foi muito difundida no meio empresarial. O Business Model Canvas consiste em um framework utilizado para descrever, analisar e projetar modelos de negócio, sendo foi aplicado e testado em organizações como IBM e Ericsson (DÍAZ-DÍAZ; MUÑOZ; PÉREZ-GONZÁLEZ, 2017). Compreende nove elementos que abrangem quatro principais áreas de um empresa: cliente, oferta, infra-estrutura e viabilidade financeira (DÍAZ-DÍAZ; MUÑOZ; PÉREZ-GONZÁLEZ, 2017). A ferramenta Business Model Canvas pode ser usada para definir modelos de negócio para produtos e serviços baseados em IoT (PACHECO; KLEIN; RIGHI, 2016).

\section{MÉTODO}

A busca de propostas de Modelos de Negócio para IoT na literatura foi desenvolvida de forma a identificar os principais e mais frequentes elementos que compõe esses modelos citados nos trabalhos acadêmicos. Foi realizada uma busca na base de dados internacional Science Direct, utilizando-se as palavras-chave Business Models e IoT, sem aspas. Quando a busca é realizada dessa forma, são identificados textos que contenham ao menos alguma das palavras inseridas, não necessariamente o termo exato. Ou seja, poderiam haver artigos que continham apenas as palavras Business, Models ou IoT. Foram identificados 28 artigos científicos que continham algumas das palavras-chave no título, palavras-chave ou abstract. Ao restringir a busca para "Business Models" e "IoT", onde a busca é realizada pelas palavras contidas exatamente entre aspas, foram identificados 13 artigos contendo as exatas palavras-chave em título, palavraschave ou abstract. Cada um dos trabalhos foi analisado com o objetivo de identificar os componentes mais frequentes em Modelos de Negócio para IoT citados nestes estudos.

Perante essa análise, foi identificado que nem todos os trabalhos encontrados apresentavam em seus estudos de fato uma proposta de Modelo de Negócio para IoT, ou seja, não apresentavam aderência aos objetivos deste estudo. Portanto, o número de casos para análise foi restrito a 12 artigos (Tabela 1).

\section{RESULTADOS}

As 12 propostas analisadas neste estudo podem ser consideradas relevantes (Tabela 1), por serem publicadas em periódicos reconhecidos e devido à abrangência da base de dados em que foram selecionados, a Science Direct. O trabalho mais frequentemente citado, a partir de 
uma análise no Google Scholar, é o de Hashem et al. (2016). O artigo foi publicado na International Journal of Information Management (IJIM), uma revista internacional de fator de impacto 2,692. Os autores abordam a expansão da tecnologia big data com a evolução da IoT e os desafios dessa aplicação em "cidades inteligentes", bem como no desenvolvimento de negócios. O segundo estudo com mais citações no meio acadêmico é o de Glova, Sabol e Vajda (2014), publicado na Procedia Economics and Finance. Os autores contextualizam as mudanças nos Modelos de Negócio com o acelerado desenvolvimento de tecnologias baseadas em Internet e faz uma análise de Modelos voltados à IoT. O trabalho de Díaz-Díaz, Muñoz e Pérez-González (2017), publicado na Future Generation Computer Systems não apresentou citação no Google Scholar dentro do período da análise (01 de junho de 2017 a 02 de junho de 2017). A revista em que foi publicado apresenta fator de impacto de 2,430. Possivelmente a falta de citações deste trabalho pode ser explicada pelo fato de ser o mais recente dentre todas as propostas analisadas.

Tabela 1 - Propostas de BM analisadas no artigo

\begin{tabular}{|c|c|c|c|}
\hline Nome & Periódico & Autor(es) & $\begin{array}{l}\text { Citações } \\
\text { GScholar }\end{array}$ \\
\hline The role of big data in smart city & $\begin{array}{l}\text { International Journal of } \\
\text { Information Management }\end{array}$ & $\begin{array}{l}\text { HASHEM et al. } \\
\text { (2016) }\end{array}$ & 28 \\
\hline $\begin{array}{c}\text { Business Models for the Internet } \\
\text { of Things Environment }\end{array}$ & $\begin{array}{l}\text { Procedia Economics and } \\
\text { Finance }\end{array}$ & $\begin{array}{l}\text { GLOVA; SABOL; } \\
\text { VAJDA. (2014) }\end{array}$ & 24 \\
\hline $\begin{array}{c}\text { Quality of Information and Access } \\
\text { Cost of IoT Resources in BPMN } \\
\text { Processes }\end{array}$ & Procedia Technology & $\begin{array}{c}\text { FERREIRA; } \\
\text { MARTINHO; } \\
\text { DOMINGOS (2014) }\end{array}$ & 8 \\
\hline $\begin{array}{l}\text { Privacy in Internet of Things: A } \\
\text { Model and Protection Framework }\end{array}$ & $\begin{array}{l}\text { Procedia Computer } \\
\text { Science } \\
\end{array}$ & $\begin{array}{c}\text { SAMANI; } \\
\text { GHENNIWA; } \\
\text { WAHAISHI. }(2015) \\
\end{array}$ & 8 \\
\hline $\begin{array}{l}\text { IOTSim: A simulator for analysing } \\
\text { IoT applications }\end{array}$ & $\begin{array}{l}\text { Journal of Systems } \\
\text { Architecture }\end{array}$ & ZENG et al. (2017) & 6 \\
\hline $\begin{array}{l}\text { Integrating wireless sensor } \\
\text { networks with statistical quality } \\
\text { control to develop a cold chain } \\
\text { system in food industries }\end{array}$ & $\begin{array}{c}\text { Computer Standards \& } \\
\text { Interfaces } \\
\end{array}$ & SHIH; WANG (2016) & 4 \\
\hline $\begin{array}{c}\text { Prototyping Business Models for } \\
\text { IoT Service }\end{array}$ & $\begin{array}{l}\text { Procedia Computer } \\
\text { Science } \\
\end{array}$ & JU; KIM; AHN (2016) & 2 \\
\hline $\begin{array}{l}\text { The Internet-of-Things: Review } \\
\text { and research directions }\end{array}$ & $\begin{array}{l}\text { International Journal of } \\
\text { Research in Marketing }\end{array}$ & $\begin{array}{c}\text { NG; WAKENSHAW } \\
(2017) \\
\end{array}$ & 2 \\
\hline $\begin{array}{l}\text { Modelos de negócio para produtos } \\
\text { e serviços baseados em internet } \\
\text { das coisas: uma revisão da } \\
\text { literatura e oportunidades de } \\
\text { pesquisas futuras }\end{array}$ & REGE-Revista de Gestão & $\begin{array}{l}\text { PACHECO; KLEIN; } \\
\text { RIGHI (2016) }\end{array}$ & 1 \\
\hline $\begin{array}{l}\text { Investigating e-business models' } \\
\text { value retention for start-ups: The } \\
\text { moderating role of venture capital } \\
\text { investment intensity }\end{array}$ & $\begin{array}{l}\text { International Journal of } \\
\text { Production Economics } \\
\end{array}$ & GUO et al. (2017) & 1 \\
\hline $\begin{array}{l}\text { Internet of Things Approach to } \\
\text { Cloud-based Smart Car Parking }\end{array}$ & $\begin{array}{l}\text { Procedia Computer } \\
\text { Science }\end{array}$ & $\begin{array}{l}\text { ATIF; DING; } \\
\text { JEUSFELD (2016) }\end{array}$ & 1 \\
\hline $\begin{array}{l}\text { Business model analysis of public } \\
\text { services operating in the smart city } \\
\text { ecosystem: The case of } \\
\text { SmartSantander }\end{array}$ & $\begin{array}{l}\text { Future Generation } \\
\text { Computer Systems }\end{array}$ & $\begin{array}{c}\text { DÍAZ-DÍAZ; } \\
\text { MUÑOZ; PÉREZ- } \\
\text { GONZÁLEZ (2017) }\end{array}$ & 0 \\
\hline
\end{tabular}

Em cada artigo analisado foi realizado o seguinte levantamento: quais os principais elementos de Modelos de Negócio voltados à IoT que são mais frequentemente citados nos trabalhos 
acadêmicos? A partir desse levantamento nas 12 propostas de Modelos de Negócio para IoT analisadas (Tabela 2), identificou-se os seguintes elementos: (i) consumidores, (ii) decisores, (iii) canais, (iv) parceiros, (v) recursos-chave, (vi) atividades-chave, (vii) proposição de valor, (viii) custos, (ix) fluxo de receita e (x) infraestrutura tecnológica.

Tabela 2 - Elementos mais frequentes em BMs para IoT

\begin{tabular}{|c|c|c|c|c|c|c|c|c|c|c|}
\hline \multicolumn{11}{|c|}{ Elementos de Business Models } \\
\hline Propostas & 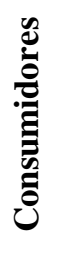 & 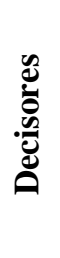 & שֶ & 苞 & 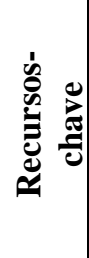 & 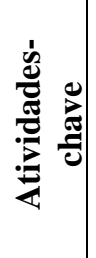 & 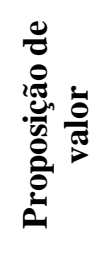 & $\stackrel{y}{n}_{\tilde{n}}^{n}$ & 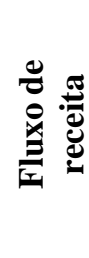 & 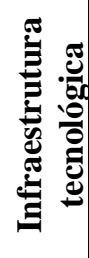 \\
\hline $\begin{array}{l}\text { HASHEM et al. } \\
(2016)\end{array}$ & $\mathrm{X}$ & $X$ & $X$ & & & $\mathrm{X}$ & & & & $\mathrm{X}$ \\
\hline $\begin{array}{c}\text { DÍAZ-DÍAZ; } \\
\text { MUÑ̃OZ; PÉREZ- } \\
\text { GONZÁLEZ (2017) }\end{array}$ & $\mathrm{X}$ & $X$ & & & $\mathrm{X}$ & $X$ & $\mathrm{X}$ & $\mathrm{X}$ & $\mathrm{X}$ & $\mathrm{X}$ \\
\hline $\begin{array}{l}\text { PACHECO; KLEIN; } \\
\text { RIGHI (2016) }\end{array}$ & $\mathrm{X}$ & $\mathrm{X}$ & $\mathrm{X}$ & & $\mathrm{X}$ & $\mathrm{X}$ & $\mathrm{X}$ & $X$ & & $\mathrm{X}$ \\
\hline $\begin{array}{c}\text { SHIH; WANG } \\
\text { (2016) }\end{array}$ & $X$ & & $X$ & & $X$ & & $X$ & $X$ & & $X$ \\
\hline $\begin{array}{l}\text { JU; KIM; AHN } \\
(2016)\end{array}$ & $\mathrm{X}$ & $\mathrm{X}$ & $\mathrm{X}$ & & $\mathrm{X}$ & $\mathrm{X}$ & $X$ & $X$ & $\mathrm{X}$ & $\mathrm{X}$ \\
\hline $\begin{array}{c}\text { FERREIRA; } \\
\text { MARTINHO; } \\
\text { DOMINGOS (2014) }\end{array}$ & & & & & $X$ & & & $X$ & & $X$ \\
\hline $\begin{array}{l}\text { GLOVA; SABOL; } \\
\text { VAJDA. (2014) }\end{array}$ & $\mathrm{X}$ & $\mathrm{X}$ & $\mathrm{X}$ & $\mathrm{X}$ & & & $\mathrm{X}$ & & & $\mathrm{X}$ \\
\hline $\begin{array}{c}\text { NG; WAKENSHAW } \\
(2017)\end{array}$ & $\mathrm{X}$ & $X$ & & & & $\mathrm{X}$ & & & $\mathrm{X}$ & $\mathrm{X}$ \\
\hline GUO et al. (2017) & & & & $\mathrm{X}$ & $\mathrm{X}$ & & $\mathrm{X}$ & $\mathrm{X}$ & & $\mathrm{X}$ \\
\hline $\begin{array}{c}\text { SAMANI; } \\
\text { GHENNIWA; } \\
\text { WAHAISHI. (2015) }\end{array}$ & $\mathrm{X}$ & $\mathrm{X}$ & & $X$ & & $X$ & & & & $\mathrm{X}$ \\
\hline ZENG et al. (2017) & $X$ & & $\mathrm{X}$ & & $\mathrm{X}$ & X & & & & $X$ \\
\hline $\begin{array}{c}\text { ATIF; DING; } \\
\text { JEUSFELD (2016) }\end{array}$ & $\mathrm{X}$ & & $\mathrm{X}$ & & & $X$ & & & & $\mathrm{X}$ \\
\hline
\end{tabular}

Em alguns dos casos analisados, os componentes citados nos Modelos de Negócio não eram expressos exatamente com as nomenclaturas apresentadas na Tabela 2. Dessa forma foi realizada uma análise sobre quais deles se referiam às mesmas definições. Um caso a ser exemplificado foi o trabalho de Guo et al. (2017) que apresenta uma proposta onde são envolvidos tamanho da equipe e experiência. Esses elementos foram aqui representados no componente Recursos-chave, que envolve todos os recursos, humanos principalmente, levados em consideração no desenvolvimento de um Modelo de Negócio para IoT.

Identificou-se que em todos os casos o componente Infraestrutura Tecnológica foi recomendado. Isso pode ser explicado pelo fato de que para a implementação da Internet das Coisas é necessária uma estrutura composta de variados componentes tecnológicos. Esses componentes estão geralmente inseridos em subsistemas definidos de acordo com as necessidades e objetivos da organização.

Outro fator identificado foi que os trabalhos que apresentaram referências de maior número de componentes em sua maioria apresentaram propostas voltadas ao Modelo de Negócio 
conhecido como Business Models Canvas. Esse modelo compreende nove elementos que abrangem quatro principais áreas de um empresa: cliente, oferta, infraestrutura e viabilidade financeira. Não há evidências que afirmem que este seja o melhor Modelo de Negócio para IoT, porém a partir desta análise foi possível identificar que este modelo pode ser utilizado para este fim.

O segundo item mais citado em Modelos de Negócio para IoT é Consumidores. Assim como em Modelos de Negócio tradicionais, o principal objetivo no desenvolvimento de novos produtos e serviços está na geração de valor dos produtos e serviços principalmente para seus clientes que, consequentemente, traz novas margens de lucro para a empresa. Portanto, verifica-se que este componente desempenha um papel importante nas definições de um novo Modelo de Negócio para as organizações.

\section{CONSIDERAÇÕES FINAIS}

O presente artigo teve por objetivo desenvolver uma análise de conteúdo dos Modelos de Negócio para IoT, para identificar os principais elementos analisados nos modelos para esse fim, a partir da realização de um compêndio dos Modelos de Negócios direcionados à IoT. Foram analisados 12 trabalhos acadêmicos sobre o tema, presentes na base de dados internacional Science Direct. Todos estes trabalhos foram publicados em revistas de relevância na área. Os resultados indicam que o componente mais frequente em Modelos de Negócio para IoT é a Infraestrutura Tecnológica, possivelmente pela necessidade de seu uso na implementação e uso da Internet das Coisas. Identificou-se ainda que os Modelos de Negócio, tanto os tradicionais quanto os voltados à IoT possuem grande foco nos consumidores, na geração de valor para o cliente. $\mathrm{O}$ valor percebido no desenvolvimento dos produtos e serviços é definido pelo valor que é entregue ao cliente final. Para trabalhos futuros sugere-se uma busca mais ampliada de propostas, que englobe maior número de bases de dados e combinações diversas de palavras-chave, uma vez que existem diferentes nomenclaturas para o tema. Sugere-se ainda a identificação dos benefícios do uso dos Modelos de Negócio para IoT e quais as principais divergências entre esses tipos de BM e os BM tradicionais. Como pesquisas futuras pode-se ainda ser destacada a possibilidade de proposta de um novo Modelo de Negócio para IoT a partir de aplicação em um caso prático, bem como os resultados alcançados com sua aplicação.

\section{REFERÊNCIAS}

AL-FUQAHA, A. et al. Internet of Things: A Survey on Enabling Technologies, Protocols, and Applications. IEEE Communications Surveys and Tutorials, v. 17, n. 4, p. 2347-2376, 2015.

ATIF, Y.; DING, J.; JEUSFELD, M. A. Internet of Things Approach to Cloud-based Smart Car Parking. Procedia Computer Science, v. 98, p. 193-198, 2016.

ATZORI, L.; IERA, A.; MORABITO, G. The internet of things: a survey. Computer Networks, v. 54, n. 15, p. 2787-2805, 2010.

BAKER, T. et al. An energy-aware service composition algorithm for multiple cloud-based 
IoT applications. Journal of Network and Computer Applications, 2017.

BORGIA, E. The internet of things vision: Key features, applications and open issues. Computer Communications, v. 54, p. 1-31, 2014.

CHAN, H. C. Y. Internet of Things Business Models. Journal of Service Science and Management, v. 8, n. August, p. 552-568, 2015.

COOPER, R. G.; KLEINSCHMIDT, E. J. New products: What separates winners from losers? The Journal of Product Innovation Management, v. 4, n. 3, p. 169-184, 1987. DÍAZ-DÍAZ, R.; MUÑOZ, L.; PÉREZ-GONZÁLEZ, D. Business model analysis of public services operating in the smart city ecosystem: The case of SmartSantander. Future Generation Computer Systems, 2017.

DIJKMAN, R. M. et al. Business models for the Internet of Things. International Journal of Information Management, v. 35, n. 6, p. 672-678, 2015.

FERREIRA, P.; MARTINHO, R.; DOMINGOS, D. Process Invariants: An Approach to Model Expected Exceptions. Procedia Technology, v. 16, p. 824-833, 2014.

GLOVA, J.; SABOL, T.; VAJDA, V. Business Models for the Internet of Things Environment. Procedia Economics and Finance, v. 15, p. 1122-1129, 2014.

GUBBI, J. et al. Internet of Things (IoT): A vision, architectural elements, and future directions. Future Generation Computer Systems, v. 29, n. 7, p. 1645-1660, 2013.

GUO, L. et al. Investigating e-business models' value retention for start-ups: The moderating role of venture capital investment intensity. International Journal of Production Economics, v. 186, n. January, p. 33-45, abr. 2017.

HASHEM, I. A. T. et al. The role of big data in smart city. International Journal of Information Management, v. 36, n. 5, p. 748-758, 2016.

JU, J.; KIM, M.-S.; AHN, J.-H. Prototyping Business Models for IoT Service. Procedia Computer Science, v. 91, p. 882-890, 2016.

LI, HUAN; XU, ZHENG-ZHONG. Research on business model of Internet of Things based on MOP. In: International Asia Conference on Industrial Engineering and Management Innovation (IEMI2012) Proceedings. Springer Berlin Heidelberg, p. 1131-1138, 2013.

MOURTZIS, D. et al. Cloud-based adaptive process planning considering availability and capabilities of machine tools. Journal of Manufacturing Systems, v. 39, p. 1-8, 2016.

NG, I. C. L.; WAKENSHAW, S. Y. L. The Internet-of-Things: Review and research directions. International Journal of Research in Marketing, v. 34, n. 1, p. 3-21, 2017. NORONHA, A. et al. Attaining IoT Value : How To Move from Connecting Things to 
Capturing Insights Gain an Edge by Taking Analytics to the Edge. Cisco, p. 19, 2014. OSTERWALDER, Alexander; PIGNEUR, Yves. Business model generation: a handbook for visionaries, game changers, and challengers. John Wiley \& Sons, 2010.

OSTERWALDER, A.; PIGNEUR, Y.; TUCCI, C. L. Clarifying business models: origins, present, and future of the concept. Communications of the Association for Information Systems, v. 15, n. 1, p. 1-43, 2005.

PACHECO, F. B.; KLEIN, A. Z.; RIGHI, R. DA R. Modelos de negócio para produtos e serviços baseados em internet das coisas: uma revisão da literatura e oportunidades de pesquisas futuras. REGE - Revista de Gestão, v. 23, n. 1, p. 41-51, 2016.

PACHECO, F. B.; KLEIN, A. Z.; RIGHI, R. DA R. Modelos de negócio para produtos e serviços baseados em internet das coisas: uma revisão da literatura e oportunidades de pesquisas futuras. REGE - Revista de Gestão, v. 23, n. 1, p. 41-51, jan. 2016.

PANG, Z. et al. Value-centric design of the internet-of-things solution for food supply chain: Value creation, sensor portfolio and information fusion. Information Systems Frontiers, v. 17, n. 2, p. 289-319, 2015.

QIN, Q.; YU, H. Research on the Internet of Things Business Model of Telecom Operators Based on the Value Net. Management \& Engineering, v. 21, n. 21, p. 350-354, 2015.

SAMANI, A.; GHENNIWA, H. H.; WAHAISHI, A. Privacy in Internet of Things: A Model and Protection Framework. Procedia Computer Science, v. 52, p. 606-613, 2015.

SHIH, C.-W.; WANG, C.-H. Integrating wireless sensor networks with statistical quality control to develop a cold chain system in food industries. Computer Standards \& Interfaces, v. 45 , p. 62-78, mar. 2016.

UCKELMANN, D.; HARRISON, M.; MICHAHELLES, F. Architecting the Internet of Things. New York: Springer, 2011.

ZENG, X. et al. IOTSim: A simulator for analysing IoT applications. Journal of Systems Architecture, v. 72, p. 93-107, jan. 2017.

ZOTT, C.; AMIT, R. Business model design: An activity system perspective. Long Range Planning, v. 43, n. 2-3, p. 216-226, 2010. 